\title{
p-TsOH catalysed KSF solid supported Michael addition with substituted isoxazoles and their reductive cyclisation to isoxazolo $[4,5-b]$ azepines
}

\author{
E. Rajanarendar*, P. Ramesh, E. Kalyan Rao, G. Mohan, and M. Srinivas \\ Department of Chemistry, Kakatiya University, Warangal-506 001 (A.P.), India \\ E-mail: eligeti_rajan@yahoo.co.in
}

\begin{abstract}
The Michael addition of substituted isoxazoles to substituted chalcones and 1,3-dicarbonyl compounds in presence of $p$-toluenesulfonic acid catalyst supported on KSF-clay proceed very efficiently and furnished the Michael adducts in excellent yields. The Michael adducts underwent reductive cyclization on treatment with $\mathrm{SnCl}_{2}-\mathrm{MeOH}$ to afford substituted isoxazolo[4,5- $b]$ azepines in high yields.
\end{abstract}

Keywords: Isoxazole Michael adducts, $p$-TsOH-KSF solid support, reductive cyclization, isoxazolo[4,5- $b]$ azepines

\section{Introduction}

Heterocycles play a vital role in pharmacological, agricultural and synthetic fields. Consequently, the development of methodologies useful for the assembly of molecules containing heterocyclic templates continues to attract the attention of both the academic and industrial communities. The investigation of the chemistry of azepines has been, and continues to be, a particularly active area of heterocyclic chemistry ${ }^{1}$.

Azepine derivatives have been found to be associated with diverse pharmacological activities such as anti-epileptic activity ${ }^{2}$, antiviral activity ${ }^{3}$, anticancer activity ${ }^{4}$, antiinsecticidal activity ${ }^{5}$, vasopressin (AVP) antagonist activity $^{6}$ and isoxazoloazepines are employed as AMPA receptors ${ }^{7}$. Similarly isoxazole nucleus can also be frequently recognized in the structure of numerous naturally occurring compounds and synthetic compounds with interesting biological and pharmacological properties ${ }^{8}$. Prompted by these observations and in continuation of our interest in designing the synthesis of biologically active fused heterocycles ${ }^{9}$, we herein report the synthesis of isoxazoloazepines bearing an additional heterocyclic substituent via Michael addition on (to) isoxazole substituents. 
The Michael addition reaction is widely recognized as one of the most important carboncarbon bond forming reactions in organic synthesis and it can generally be carried out with a strong base ${ }^{10}$. However, the base catalysed method sometimes suffers from disadvantages of incompatibility with base-sensitive functionality and the occurrence of other side reactions, such as autocondensations and retro-Michael type decompositions. When we carried out the Michael addition on isoxazole derivatives in presence of $\mathrm{Et}_{3} \mathrm{~N}^{11}$, the reaction required long heating periods and the product yields were only moderate and unwanted side products were formed ${ }^{12}$. To circumvent such problems, considerable attention has recently been focused on the use of phase transfer catalysts ${ }^{13}$, transition metal complexes ${ }^{13}$, Lanthanides ${ }^{14}$, alumina and clay supported catalysts ${ }^{15}$ and Lewis acid catalysts such as $\mathrm{BF}_{3} \cdot \mathrm{Et}_{2} \mathrm{O}^{16 \mathrm{a}}, \mathrm{Yb}(\mathrm{OTf})_{3}{ }^{16 \mathrm{~b}}, \mathrm{Bi}(\mathrm{OTf})_{3}{ }^{16 \mathrm{c}}$, $\mathrm{ZrCl}_{4}{ }^{16 \mathrm{~d}}$ and trifluoromethane sulfonic acid $^{17}$ in Michael additions.

Although these methods are suitable for certain synthetic applications, many of these procedures are associated with one (or more) disadvantages such as expensive or toxic reagents, long reactions time, tedious workup and low yeilds Thus, development of new methods using cheap and commercially available less toxic reagents to afford high yields of products in short reaction times are important.

\section{Results and Discussion}

In a sequel to our work ${ }^{18}$ for the development of new methods of Michael addition reactions, we describe a mild and efficient protocol for the addition of 3,5-dimethyl-4-nitroisoxazoles ${ }^{19}$ to $\alpha, \beta$ unsaturated ketones and addition of 1,3-dicarbonyls to 3-methyl-4-nitro-5-styrylisoxazoles using catalytic amount of $p$-TsOH adsorbed on KSF solid support under solvent-free conditions to generate the Michael adducts very efficiently in excellent yields, in which the isoxazole serves as a Michael donor or Michael acceptor.

The synthesis of isoxazolo[4,5-b]azepines consists of two steps: 1) synthesis of Michael adducts, and 2) reductive cyclisation of Michael adducts to isoxazoloazepines.

In the first step we have studied the Michael addition of 3,5-dimethyl-4-nitroisoxazole (Michael donor) to substituted chalcones (Michael acceptor) and also the addition of 1,3dicarbonyl compounds (Michael donor) to substituted styrylisoxazole (Michael acceptor) using 2 mol \% of $p$-TsOH on KSF solid support under solvent-free conditions (Scheme-1). The reaction is very fast and to our satisfaction, the addition product was obtained in almost quantitative yield in $2 \mathrm{~h}$. It seems that $p$-TsOH-KSF solid support is a much better alternative to effect the Michael reaction in terms of better yields ( $85 \%)$ and short reaction times $(2 \mathrm{hr})$.

Encouraged by these results, several chalcones and aldehydes were examined under different conditions. The best results were achieved when the reactions were carried out at $120^{\circ} \mathrm{C}$ for 0.5 - $2 \mathrm{~h}$. in presence of a catalytic amount of $p-\mathrm{TsOH}$. The scope and generality of this transformation is illustrated with different Michael donors and acceptors and results are summarized in Table 1. 
Table 1. Synthesis of isoxazolyl Michael adducts catalysed by $p$-TsOH-KSF solid support under solvent free conditions

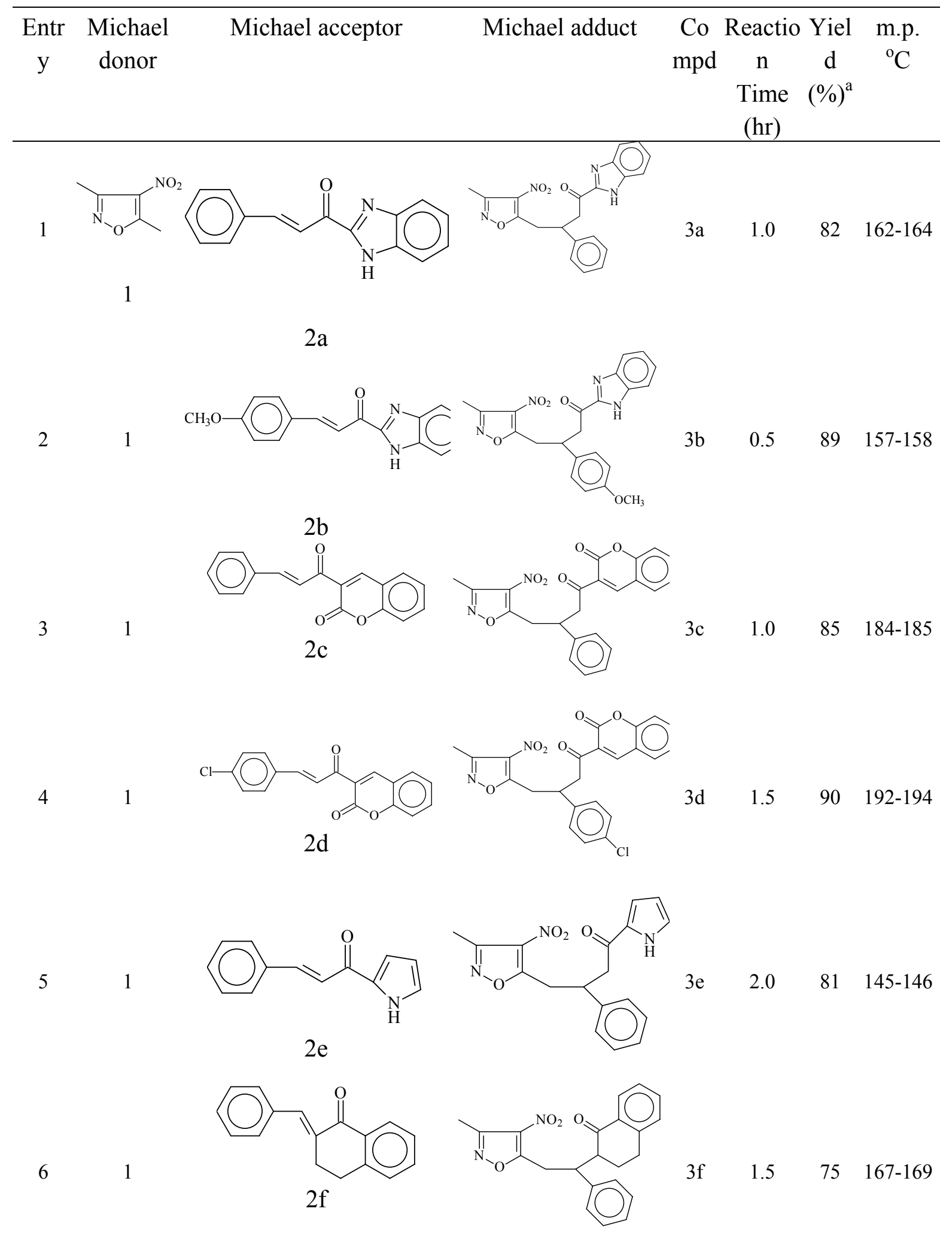


8<smiles>CC(=O)CC(=O)CCl</smiles>

$4 b$

9<smiles>CC(=O)CC(=O)C(F)(F)F</smiles>

$4 c$

10

$4 a$<smiles>Cc1noc(/C=C/c2ccc[nH]2)c1[N+](=O)[O-]</smiles>

$5 b$

11

$4 b$

12

$4 \mathrm{c}$

$5 b$
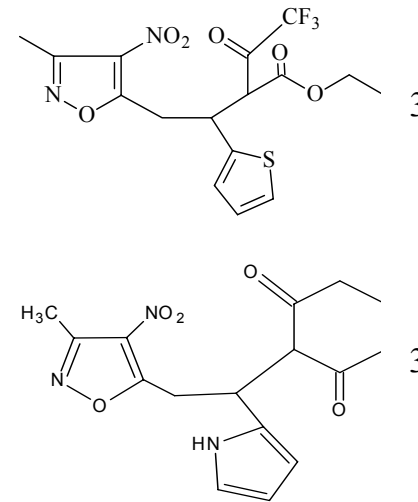

$3 \mathrm{j} \quad 1.5 \quad 78 \quad 224-227$

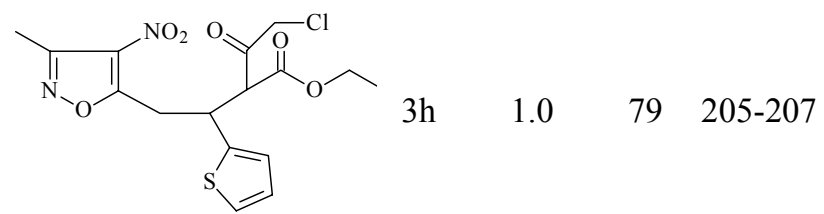

$0.5 \quad 92 \quad 180-182$

$1.5 \quad 78 \quad 224-227$
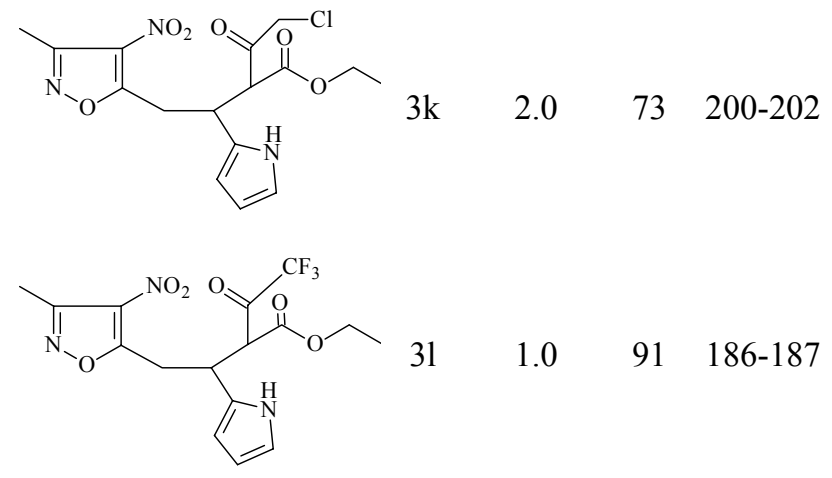

${ }^{a}$ Isolated yield. All the compounds were characterized by mp, spectral (IR, ${ }^{1} \mathrm{H}$ NMR and Mass), and analytical data. 

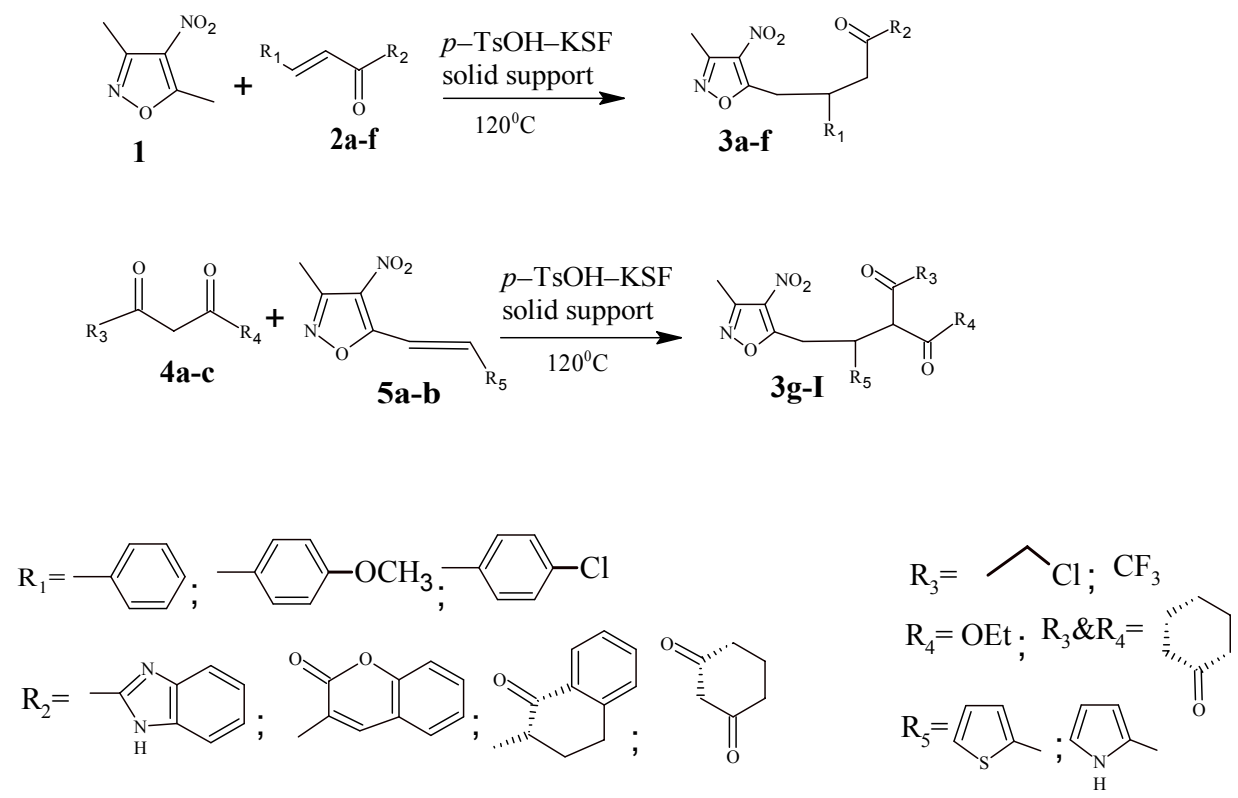

\section{Scheme 1}

In the subsequent step, we have performed reductive cyclisation of Michael adducts (3) to isoxazoloazepines (5). Michael adduct (3a) $(1 \mathrm{mmol})$, was heated with $\mathrm{SnCl}_{2}(5 \mathrm{mmol})$ in methanol $(10 \mathrm{ml})$ for $4 \mathrm{hr}$ to afford the corresponding isoxazoloazepine in $90 \%$ yield (Scheme 2). The process involves reduction-cyclization to give title compounds. To our satisfaction, the reductive cyclisation resulted in high yields.

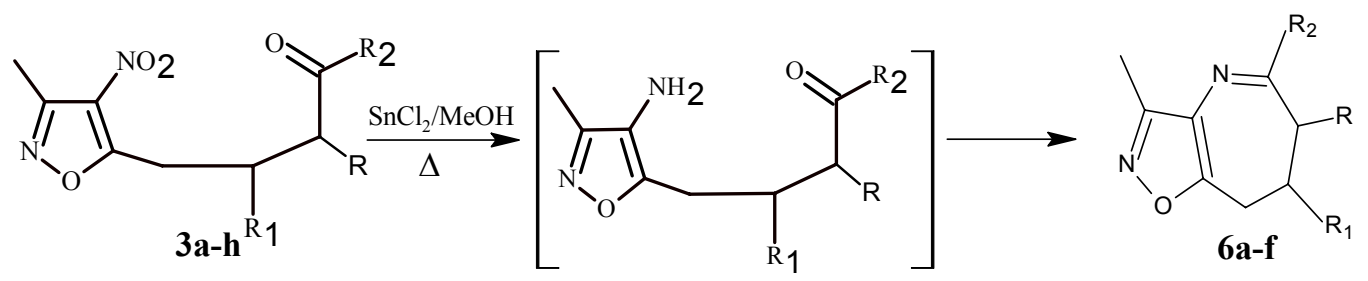

\section{$\mathbf{R}=\mathbf{H}$, acyl}

\section{Scheme 2}

Encouraged by this result, the reductive cyclisation of all Michael adducts (Table-1) to isoxazoloazepines (6) was accomplished in 4-7 h. and in high yield (70-90\%) by heating in $\mathrm{SnCl}_{2} / \mathrm{MeOH}$. The results are summarized in Table 2.

In conclusion, we have demonstrated the synthesis of isoxazoloazepines via Michael addition followed by reductive cyclisation. For Michael addition, we have developed a convenient and highly efficient protocol by using $p$-TsOH adsorbed on KSF solid support under solvent-free conditions with a variety of Michael donors and acceptors. We believe that the method offers considerable advantages for producing several types of Michael adducts in view of 
its high efficiency, operational simplicity and convenient work-up procedure and easily accessible $p$-TsOH adsorbed on KSF solid support as the catalyst makes methodology of wide synthetic applicability and commercial utility.

Table 2. Synthesis of substituted isoxazoloazepines by reductive cyclization

\begin{tabular}{|c|c|c|c|c|c|c|}
\hline Entry & $\begin{array}{l}\text { Michael } \\
\text { Adduct }\end{array}$ & Substituted isoxazolo azepines & Compd & $\begin{array}{c}\text { Reaction } \\
\text { time } \\
(\mathrm{hr})\end{array}$ & $\begin{array}{l}\text { Yield } \\
(\%)^{\mathrm{a}}\end{array}$ & $\begin{array}{c}\mathrm{m} . \mathrm{p} \\
{ }^{\circ} \mathrm{C}\end{array}$ \\
\hline 1 & $3 a$ & & $6 a$ & 4 & 79 & $153-155$ \\
\hline 2 & $3 b$ & & $6 b$ & 5 & 72 & $140-142$ \\
\hline 3 & $3 c$ & & $6 c$ & 7 & 85 & $171-173$ \\
\hline 4 & $3 d$ & & $6 \mathrm{~d}$ & 6 & 81 & $177-178$ \\
\hline 5 & $3 e$ & & $6 e$ & 5 & 79 & $134-137$ \\
\hline
\end{tabular}


6

$3 f$

$7 \quad 3 g$

8

$3 \mathrm{~h}$

$9 \quad 3 \mathrm{i}$
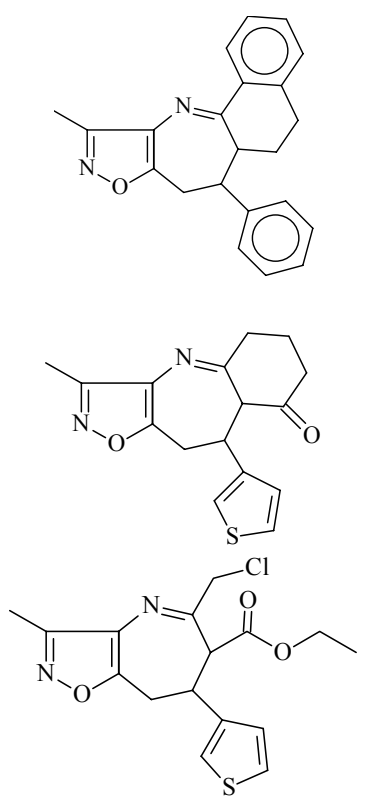

$6 f$

7

74

148-150

$6 g$

6

$70 \quad 186-187$

$6 \mathrm{~h}$

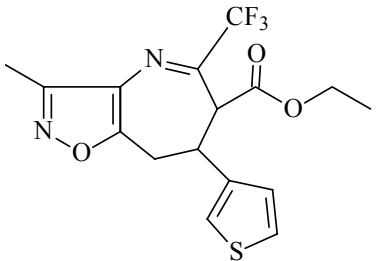

$6 \mathrm{i}$<smiles>Cc1noc2c1N=C1CCCC(=O)C1C(c1ccc[nH]1)C2</smiles>

10

$3 \mathrm{j}$

11

$3 \mathrm{k}$

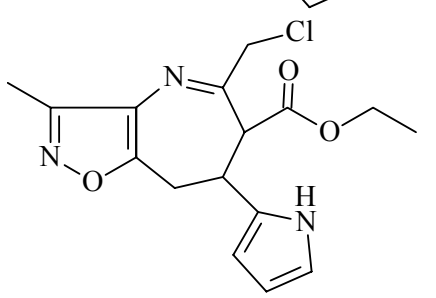

$6 j$

6

$72 \quad 214-215$

$6 \mathrm{k}$

4

$84 \quad 189-190$

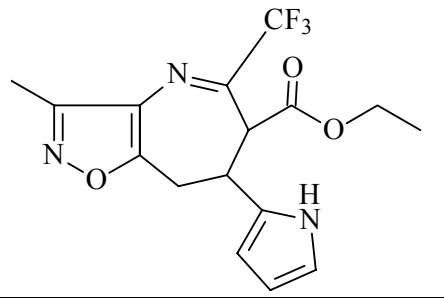

61

5

$89 \quad 175-176$

12

31 


\section{Experimental Section}

Typical experimental procedure for the synthesis of substituted Michael adducts A mixture of 3,5-dimethyl-4-nitro isoxazole (donor) $(10 \mathrm{mmol})$ and substituted chalcone (acceptor) (10 mmol) or 1,3-dicarbonyl compounds (donor) $(10 \mathrm{mmol})$ and substituted styryl isoxazoles (acceptor) $10 \mathrm{mmol}$ were taken in dichloromethane and added $p$ - $\mathrm{TsOH}$ ( 2 mole \%) than adsorbed on montmorillonite-KSF clay (1 gr) and allowed to fuse for $0.5-2 \mathrm{hr}$ at $120^{\circ} \mathrm{C}$ in an oil bath. After completion of the reaction (monitored with TLC), it was allowed to cool. The solid mass was triturated with water and dried than taekn in a column with short plug of silica gel and eluted with ethyl acetate and n-hexane (1:9). Evaporation of solvent furnished Michael adducts (3a-l) in $73-92 \%$ yields.

Compound 3b. Pale yellow crystalline solid, m.p. 162-164 ${ }^{\circ} \mathrm{C}$. IR $(\mathrm{KBr}): 1685,3250 \mathrm{~cm}^{-1}$. ${ }^{1} \mathrm{H}$ NMR $\left(200 \mathrm{MHz}, \mathrm{CDCl}_{3}\right): 2.3\left(\mathrm{~s}, 3 \mathrm{H}, \mathrm{CH}_{3}\right), 3.3\left(\mathrm{~m}, 2 \mathrm{H},-\mathrm{CH}_{2}-\mathrm{CH}\right), 3.6\left(\mathrm{~s}, 3 \mathrm{H}, \mathrm{OCH}_{3}\right), 4.0(\mathrm{~m}$, $\left.1 \mathrm{H},-\mathrm{CH}_{2}-\mathrm{CH}\right), 4.3\left(\mathrm{~m}, 2 \mathrm{H}, \mathrm{CH}_{2}-\mathrm{CO}\right), 7.0-7.9(\mathrm{~m}, 8 \mathrm{H}, \mathrm{Ar}-\mathrm{H}), 10.0\left(\mathrm{bs}, 1 \mathrm{H}, \mathrm{NH}, \mathrm{D}_{2} \mathrm{O}\right.$ exchangeable). MS (EI) : m/z $421\left[\mathrm{M}^{+}+1\right]$, Anal. Cald. for $\mathrm{C}_{22} \mathrm{H}_{20} \mathrm{~N}_{4} \mathrm{O}_{5}: \mathrm{C}, 62.85 ; \mathrm{H}, 4.76 ; \mathrm{N}$, 13.13. Found: C, 62.81; H, 4.69; N, 13.19\%.

Compound 3d. Yellowish crystalline solid, m.p. 192-194 ${ }^{\circ} \mathrm{C}$. IR (KBr) : 1698, 1725, $3325 \mathrm{~cm}^{-1}$. ${ }^{1} \mathrm{H}$ NMR (200 MHz, $\left.\mathrm{CDCl}_{3}\right): 2.2\left(\mathrm{~s}, 3 \mathrm{H}, \mathrm{CH}_{3}\right), 3.2\left(\mathrm{~m}, 2 \mathrm{H}, \mathrm{CH}_{2}-\mathrm{CH}\right), 3.8\left(\mathrm{~m}, 1 \mathrm{H}, \mathrm{CH}_{2}-\mathrm{CH}\right), 4.4$ (m, 2H, $\left.\mathrm{CH}_{2}-\mathrm{CO}\right), 6.1$ (s, coumarin-H), 7.1-7.8 (m, 8H, Ar-H), 10.4 (bs, 1H, NH, $\mathrm{D}_{2} \mathrm{O}$ exchangeable). MS (EI) : m/z $452\left[\mathrm{M}^{+}\right]$, Anal. Cald. for $\mathrm{C}_{23} \mathrm{H}_{17} \mathrm{~N}_{2} \mathrm{O}_{6} \mathrm{Cl}: \mathrm{C}, 61.06 ; \mathrm{H}, 3.76 ; \mathrm{N}$, 6.19. Found: C, 61.12; H, 3.71; N, 6.15\%.

Compound 3h. White crystalline solid, m.p. 205-207 ${ }^{\circ}$ C. IR (KBr) : 1705, $1728 \mathrm{~cm}^{-1}$. ${ }^{1} \mathrm{H}$ NMR $\left(200 \mathrm{MHz}, \mathrm{CDCl}_{3}\right): 0.9$ (t, 3H, $\left.-\mathrm{CH}_{2}-\mathrm{CH}_{3}\right), 2.2\left(\mathrm{~s}, 3 \mathrm{H}, \mathrm{CH}_{3}\right), 3.2\left(\mathrm{~m}, 2 \mathrm{H}, \mathrm{CH}_{2}-\mathrm{CH}\right), 3.8(\mathrm{~m}, 1 \mathrm{H}$, $\left.\mathrm{CH}_{2} \mathrm{C}-\mathrm{CH}\right), 4.2\left(\mathrm{q}, 2 \mathrm{H}, \mathrm{OCH}_{2}-\mathrm{CH}_{3}\right), 4.7$ (s, 2H, $\left.-\mathrm{COCH}_{2}-\mathrm{Cl}\right), 4.9$ (d, 1H, CH-CO), $6.9-7.2(\mathrm{~m}$, $3 \mathrm{H}$, thiophene-H), MS (EI) : m/z $400\left[\mathrm{M}^{+}\right]$, Anal. Cald. for $\mathrm{C}_{16} \mathrm{H}_{17} \mathrm{~N}_{2} \mathrm{O}_{6} \mathrm{SCl}$ : C, 48.00; H, 4.25; N, 7.00; Found: C, 48.08; H, 4.09; N, 7.05\%.

Compound 31. White crystalline solid, m.p. $186-187^{\circ} \mathrm{C}$. IR (KBr) : 1690, 1721, $3340 \mathrm{~cm}^{-1} .{ }^{1} \mathrm{H}$ NMR $\left(200 \mathrm{MHz}, \mathrm{CDCl}_{3}\right): 1.1\left(\mathrm{t}, 3 \mathrm{H}, \mathrm{O}-\mathrm{CH}_{2} \mathrm{CH}_{3}\right), 2.3\left(\mathrm{~s}, 3 \mathrm{H}, \mathrm{CH}_{3}\right), 3.3\left(\mathrm{~m}, 2 \mathrm{H}, \mathrm{C}_{4}-\mathrm{H}\right), 3.7(\mathrm{~m}$, $\left.1 \mathrm{H}, \mathrm{C}_{3}-\mathrm{H}\right), 4.3$ (q, 2H, -O- $\left.\mathrm{CH}_{2}-\mathrm{CH}_{3}\right), 4.8$ (d, 1H, CH-CO), 6.7-7.2 (m, 3H, pyrrole-H), 9.8 (bs, $\mathrm{NH}, \mathrm{D}_{2} \mathrm{O}$ exchangeable), MS (EI) : $\mathrm{m} / \mathrm{z} 403\left[\mathrm{M}^{+}\right]$, Anal. Cald. for $\mathrm{C}_{16} \mathrm{H}_{16} \mathrm{~N}_{3} \mathrm{O}_{6} \mathrm{~F}_{3}: \mathrm{C}, 47.64 ; \mathrm{H}$, 3.97; N, 10.42. Found: C, 47.71; H, 3.92; N, 10.39\%.

\section{General procedure for the synthesis of substituted isoxazolo azepines}

The substituted Michael adducts $(10 \mathrm{mmol})$ and $\mathrm{SnCl}_{2} \cdot 2 \mathrm{H}_{2} \mathrm{O}(100 \mathrm{mmol})$ were dissolved in methanol $(25 \mathrm{ml})$ and refluxed for $4-7 \mathrm{hr}$. After completion of the reaction (monitored with TLC), solvent was removed in vacuo. The solid mass was decomposed with cold water and the reaction solution was carefully adjusted to $\mathrm{pH} 8$ with $10 \% \mathrm{NaHCO}_{3}$ solution and then extracted with ethyl acetate $(2 \times 30 \mathrm{ml})$. The combined organic layers were dried over $\mathrm{Na}_{2} \mathrm{SO}_{4}$ and evaporated under vaccum and purified by column chromatography. Elution with $\mathrm{MeOH}$ and nhexane in 2:8 ratio afforded substituted isoxazoloazepines (6a-1) in 72-90\% yields. 
Compound 6b. Yellow crystalline solid, m.p. $153-155^{\circ} \mathrm{C}$. IR $(\mathrm{KBr}): 1610,3365 \mathrm{~cm}^{-1} \cdot{ }^{1} \mathrm{H}$ NMR $\left(200 \mathrm{MHz}_{\mathrm{CDCl}}\right): 2.2\left(\mathrm{~s}, 3 \mathrm{H}, \mathrm{CH}_{3}\right), 3.2\left(\mathrm{~m}, 2 \mathrm{H}, \mathrm{CH}_{2}-\mathrm{CH}\right), 3.6\left(\mathrm{~s}, 3 \mathrm{H}, \mathrm{OCH}_{3}\right), 3.8(\mathrm{~m}, 1 \mathrm{H}$, $\left.\mathrm{CH}_{2}-\mathrm{CH}\right), 4.1$ (m, 2H, CH $\left.2-\mathrm{CO}\right), 6.9-7.8$ (m, 8H, Ar-H), 9.9 (bs, 1H, NH, $\mathrm{D}_{2} \mathrm{O}$ exchangeable). MS (EI) : m/z $372\left[\mathrm{M}^{+}\right]$, Anal. Cald. for $\mathrm{C}_{22} \mathrm{H}_{20} \mathrm{~N}_{4} \mathrm{O}_{2}$ : C, 70.96; H, 5.37; N, 15.05. Found: C, $70.91 \mathrm{H}, 5.42, \mathrm{~N}, 15.14 \%$.

Compound 6d. Yellow crystalline solidm, m.p. 177-178. IR (KBr) : $16151727,3310 \mathrm{~cm}^{-1}$. ${ }^{1} \mathrm{H}$ NMR (200 MHz, $\left.\mathrm{CDCl}_{3}\right): 2.1\left(\mathrm{~s}, 3 \mathrm{H}, \mathrm{CH}_{3}\right), 3.1\left(\mathrm{~m}, 2 \mathrm{H}, \mathrm{CH}_{2}-\mathrm{CH}\right), 3.6\left(\mathrm{~m}, 1 \mathrm{H}, \mathrm{CH}_{2}-\mathrm{CH}\right), 4.3$ $\left(\mathrm{m}, 2 \mathrm{H}, \mathrm{CH}_{2}-\mathrm{C}=\mathrm{N}\right), 6.3$ (s, coumarin-H), 7.0-7.8 (m, 8H, Ar-H), 10.5 (bs, $1 \mathrm{H}, \mathrm{NH}_{2} \mathrm{D}_{2} \mathrm{O}$ exchangeable). MS (EI) : m/z $404\left[\mathrm{M}^{+}\right]$, Anal. Cald. for $\mathrm{C}_{23} \mathrm{H}_{17} \mathrm{~N}_{2} \mathrm{O}_{3} \mathrm{Cl}: \mathrm{C}, 68.31 ; \mathrm{H}, 4.20 ; \mathrm{N}$, 6.93. Found: C, 68.39; H, 4.12; N, 6.99\%.

Compound 6h. White crystalline solid, m.p. $200-201^{\circ} \mathrm{C}$. IR (KBr) : 1606, $1718 \mathrm{~cm}^{-1}$. ${ }^{1} \mathrm{H}$ NMR $\left(200 \mathrm{MHz}, \mathrm{CDCl}_{3}\right): 1.0\left(\mathrm{t}, 3 \mathrm{H},-\mathrm{OCH}_{2} \mathrm{CH}_{3}\right), 2.2\left(\mathrm{~s}, 3 \mathrm{H}, \mathrm{CH}_{3}\right), 3.1\left(\mathrm{~m}, 2 \mathrm{H}, \mathrm{C}_{8}-\mathrm{H}\right), 3.7(\mathrm{~m}, 1 \mathrm{H}$, $\left.\mathrm{CH}_{2}-\mathrm{CH}\right), 4.3\left(\mathrm{q}, 2 \mathrm{H},-\mathrm{OCH}_{2} \mathrm{CH}_{3}\right), 4.6\left(\mathrm{~s}, 2 \mathrm{H},-\mathrm{CO}-\mathrm{CH}_{2}-\mathrm{Cl}\right), 4.8(\mathrm{~d}, 1 \mathrm{H}, \mathrm{CH}-\mathrm{C}=\mathrm{N}), 6.8-7.3(\mathrm{~m}$, $3 \mathrm{H}$, thiophene-H). MS (EI) : m/z $352\left[\mathrm{M}^{+}\right]$, Anal. Cald. for $\mathrm{C}_{16} \mathrm{H}_{17} \mathrm{~N}_{2} \mathrm{O}_{3} \mathrm{ClS}: \mathrm{C}, 63.15 ; \mathrm{H}, 5.59$; N, 9.21. Found: C, 63.18; H, 5.52; N, 9.30\%.

Compound 61. White crystalline solid, m.p. $175-176^{\circ} \mathrm{C}$. IR $(\mathrm{KBr}): 1621,1724,3375 \mathrm{~cm}^{-1}$. ${ }^{1} \mathrm{H}$ NMR $\left(200 \mathrm{MHz}, \mathrm{CDCl}_{3}\right): 1.2\left(\mathrm{t}, 3 \mathrm{H}, \mathrm{O}-\mathrm{CH}_{2} \mathrm{CH}_{3}\right), 2.2\left(\mathrm{~s}, 3 \mathrm{H}, \mathrm{CH}_{3}\right), 3.1\left(\mathrm{~m}, 2 \mathrm{H}, \mathrm{CH}_{2}-\mathrm{CH}\right), 3.5$ $\left(\mathrm{m}, 1 \mathrm{H}, \mathrm{CH}_{2}-\mathrm{CH}\right), 4.2$ (q, 2H, O- $\left.\mathrm{CH}_{2} \mathrm{CH}_{3}\right), 4.7(\mathrm{~d}, \mathrm{CH}-\mathrm{C}=\mathrm{N}), 6.6-7.1(\mathrm{~m}, 3 \mathrm{H}$, pyrrole-H), 9.9 (bs, NH, $\mathrm{D}_{2} \mathrm{O}$ exchangeable), MS (EI) : m/z $355\left[\mathrm{M}^{+}\right]$, Anal. Cald. for $\mathrm{C}_{16} \mathrm{H}_{16} \mathrm{~N}_{3} \mathrm{O}_{3} \mathrm{~F}_{3}: \mathrm{C}, 54.08$; H, 4.50; N, 11.80. Found: C, 54.15; H, 4.52; N, 11.69\%.

\section{References}

1. Le Count, D. J. Comprehensive Heterocyclic Chemistry II Katritzky, A. R.; Rees, C. W.; Scriven, E. F. V. Eds., Pergamon: Oxford, 1996; Vol 9; pp 1-44 .

2. Patton, J. R.; Dudbey, K. H. J. Heterocycl. Chem. 1979, 16, 257.

3. Stamatian, G.; George, B.; Foscolos, G. F.: Kolocouris, A.; Koplocouris, N.; Paunecouque, C.; Witvrouw, M.; Padalko, E.; Neyts, J.; De Clercq, E. Bioorg. Med. Chem. Lett.,2003, 5485 .

4. Smith III, A. B.; Young, S. C..; Pettit, R. G.; Hirschmann, R. Tetrahedron 2003, 6991.

5. Kattenegger, E.; Brem, B.; Mereiter, K.; Kalchhauser, H.; Kahlig, H.; Hofer, O.; Vijrodaya, S.; Gregar, H. Phytochemistry 2003, 803.

6. Albright, D. J.; Efren, G.; Santos, D.; Jhan, P.; Dusza, P.; Chan, S.: Caupet, J.; Xun Ru.; Mazandariain, H. Bioorg. Med. Chem. Lett. 2000, 695.

7. Madieu, U.; Frolund, B.; Iund, T. M.; Ebert, B.; Krogsgaard-Larsen, P. Eur. J. Med. Chem. 1993, 791.

8. (a) Barot, V. M.; Patel, M. R.; Naik, H. B. Asian J. Chem. 2001, 13, 341. (b) BangAnderson, B.; Ahmadian, H.; Lenz, S. M.; Stensbol, T. B.; Madsen, U.; Bogeso, K. P.; Krogsgaard-Larsen, P. J. Med. Chem. 2000, 43, 4910. (c) Kusumi, T.; Chang, C. C.; 
Wheeler, M.; Kubo, I.; Naganishi, K.; Naoki, H. Tetrahedron Lett. 1981, 22, 3451. (d) Murthy, A. K.; Rao, K. S. R. K. M.; Rao, N. S.V.; J. Indian Chem. Soc. 1976, 1047.

9. (a) Rajanarendar, E.; Srinivas, M.; Ramu, K. Synth. Commun. 2003, 33, 3077. (b) Rajanarendar, E.; Mohan, G.; Ramesh, P.; Karunakar, D. Tetrahedron Lett., 2006, 47, 4957. c) Rajanarendar, E.; Ramu, K.; Kalyan Rao, E.; Ind. J. Heterocycl. Chem. 2005, 15, 23. d) Rajanarendar, E.; Srinivas, M.; Ramesh, P.; Ramu, K. Ind. J. Chem., 2005, 44B, 1927.

10. Bergman, E. D.; Ginsburg, D.; Pappo, R. Org. React. 1959, 10, 595.

11. (a) Rao, C. J.; Murthy, A. K. Indian J. Chem. 1978, 16B, 636. (b) Rao, C. J.; Reddy, K. M., Murthy, A. K. Indian J. Chem. 1981, 20B, 997. (c) Reddy, K. M.; Rao, C. J.; Murthy, A. K. Indian J. Chem. 1985, 24B, 212.

12. Adamo, M. F. A.; Chimichi, S.; De Sio, F.; Donati, D.; Sauti-Fantani, P. Tetrahedron Lett. 2002, 43, 4157.

13. Rele, D. N.; Trivedi, G. K. J. Scientific \& Ind. Res. 1993, 52, 13.

14. Vas Westrenen, J.; Roggen, R. M.; Hoefnagel, M. A.; Peters, J. A.; Keiboom, A. P. G.; Van Bekkum, H. Tetrahedron 1990, 46, 5741.

15. (a) Clark, J. H.; Cork, D. G.; Robertson, M. S. Chem. Lett. 1983, 1145. (b)Laszio, P.; Pennetreau, P. Tetrahedron Lett. 1985, 26, 2645.

16. (a) Sato, T.; Wakahara, Y.; Otera, J.; Nazaki, H. Tetrahedron 1991, 47, 9773. (b) Keller, E.; Feringa, B. L. Tetrahedron Lett. 1996, 37, 1879. c) Ravivarala, M.; Alam, M.; Adapa, S. R. Synlett 2003, 5, 720. (d) Smitha, G.; Patnaik, S.; Sanjeeva Reddy, C. Synthesis 2005, 55,711 .

17. Kotsuki, H.; Koji-Arimura, Ohishi, T.; Maruzasa, R. J. Org. Chem. 1999, 64, 3770.

18. (a) Rajanarendar, E.; Ramu, K.; Karunakar, D.; Ramesh, P. J. Heterocycl. Chem. 2005 42, 711. (b) Rajanarendar, E.; Ramesh, P.; Srinivas, M.; Ramu, K.; Mohan, G. Synth. Commun. 2006, 36, 665. (c) Rajanarendar, E.; Karunakar, D.; Ramesh, P.; Kalyan Rao, E.; Hetrocyclic Commun. 2006, 12, 355. (d) Rajanarendar, E.; Mohan, G.; Ramesh, P.; Srinivas, M. J. Heterocycl. Chem. 2007, 44, 215.

19. Musante, C.; Berretti, R. Gazz. Chim. Ital. 1949, 79, 683. 